\title{
Tracking a moving boundary layer transition front in supersonic flow using infrared thermography
}

\author{
Rogier Giepman ${ }^{1}$, Ferry Schrijer and Bas van Oudheusden \\ Aerospace Engineering Department, Delft University of Technology, Kluyverweg 1, 2629 HS Delft, The Netherlands
}

\begin{abstract}
Infrared thermography is used to determine the instantaneous transition location of a supersonic flat plate boundary layer. In order to have sufficient contrast for the IR measurements a stainless steel plate is uniformly heated before the wind tunnel is started. During the run the plate cools down rapidly and the decreasing wall temperature stabilises the laminar boundary layer, causing a moving transition front. A linear relation between the transition location and wall temperature is found. The obtained results agree well with surface oil flow measurements that were carried out on a cold plate.
\end{abstract}

\section{Introduction}

Boundary layer transition plays an important role in the design of high-speed flight vehicles. Not only does it affect the amount of skin friction drag, it also has a profound effect on the heat loads that the structure of such a vehicle has to withstand. A turbulent boundary layer has a much higher convective surface heat flux compared to a laminar one and therefore will cause the structure to rapidly heat up and achieve high temperatures. The exact location where transition occurs is difficult to predict since it depends on a multitude of parameters such as the free stream conditions (Reynolds number, Mach number and free stream noise level) and the surface roughness. Therefore it is of prime importance to have a means to accurately detect the transition location. Oil flow visualization is often used for this purpose whereby the thickness of the residual oil film (generally mixed with some colouring agent such as $\mathrm{TiO}_{2}$ ) is inversely proportional to the skin friction. This approach requires the application of oil on the surface and in practise it is very likely that small irregularities present in the oil cause premature transition effectively making it unsuitable for this purpose. An alternative technique that is often used in compressible flows is to detect transition by means of spark light schlieren/shadowgraphy. Although this technique is truly non-intrusive, it also is purely two dimensional in the sense that the measurement integrates along the optical path making it unable to detect span-wise variations in the transition front. An alternative to the above mentioned techniques is infrared thermography through which the location of boundary layer transition is detected by measuring the surface temperature. The technique does not rely on the use of any surface coating and is capable of measuring span-wise variations in the transition location.

When applying IR thermography in a wind tunnel it is quite common to heat up the model before the run to increase the thermal contrast between the laminar and turbulent part of the flow. Since the convective heat flux is directly proportional to the temperature difference between the model and the adiabatic wall temperature. A larger temperature difference in combination with a large heat transfer coefficient (turbulent flow) causes a larger convective heat flux and thus more pronounced temperature variations with respect to a low heat transfer coefficient (laminar flow). Although the heating of a wind tunnel model increases the measurability of the phenomenon, it is a well-known fact that a hot wall promotes transition due to the amplification of Tollmien-Schlichting waves [1]. So, when during a wind tunnel test, the temperature of the model decreases, it can be expected that the transition location moves downstream.

In this study, infrared thermography measurements are used to track such a moving transition front on a flat plate during a wind tunnel run in supersonic flow $(M=1.7)$. The experimental setup used for these measurements is discussed in section 2, the data reduction techniques are treated in section 3 and the results of this study are presented in section 4.

\section{Experimental setup}

The experiments were carried out in the TST-27 blow-down transonic / supersonic wind tunnel of the Delft University of Technology. The test section measures $270 \times 280 \mathrm{~mm}^{2}$ and the tunnel was operated at Mach 1.7 , a total pressure of 2.3 bar and a total temperature of approximately $278 \mathrm{~K}$. This resulted in a freestream velocity of $452 \mathrm{~m} / \mathrm{s}$ and a unit Reynolds number of $3510^{6}$. The freestream turbulence level is approximately $0.5 \%$ of the freestream velocity for these specific operating conditions. To investigate the effects of the Reynolds number on the transition location, also one run was performed at a total pressure of 4.6 bar, which corresponds to a unit Reynolds number of $70 \quad 10^{6}$.

The infrared thermography measurements were performed on a partial-span flat plate, which was mounted vertically in the center of the test section (see Fig.1). The model was manufactured out of stainless steel T316, which was spray-painted black to increase its emissivity. The stainless steel has a density of $=8000 \mathrm{~kg} / \mathrm{m}^{3}$, a thermal conductivity of $k=16.2 \mathrm{~W} / \mathrm{mK}$ and a specific heat capacity $c$ of $500 \mathrm{~J} / \mathrm{kgK}$, which results in a thermal product of $c k=$ $64.8 \cdot 10^{6} \mathrm{~J}^{2} \mathrm{~m}^{-4} \mathrm{~K}^{-2} \mathrm{~s}^{-1}$. This value is two orders of magnitude higher than that of a typical polycarbonate material (for

\footnotetext{
${ }^{1}$ PhD student, Aerodynamics, RH.M.Giepman@tudelft.nl
} 
example Makrolon has a thermal product of $c k=0.3 \cdot 10^{6} \mathrm{~J}^{2} \mathrm{~m}^{-4} \mathrm{~K}^{-2} \mathrm{~s}^{-1}$ ) and in general a low thermal product is beneficial, because it maximizes the output temperature signal for a given surface heat flux. Unfortunately, polycarbonate materials do not meet the structural and aerodynamic requirements and therefore it was necessary to construct the model out of a stainless steel, which is strong enough but also has a relatively large thermal product and low conductivity (compared to other metallic materials).

The measurements were performed with a CEDIP Titanium 530L infrared camera, which uses a MCT sensor having $320 \times 236$ pixels. The camera is sensitive in the spectral range of $7.7-9.3 \mathrm{~m}$. A Stirling cycle is used to cool the sensor, which brings down the noise equivalent temperature difference (NETD) to $25 \mathrm{mK}$. An integration time of $340 \mathrm{~s}$ was used and images were recorded at a frequency of $25 \mathrm{~Hz}$. The camera was equipped with a $25 \mathrm{~mm}$ objective, delivering a spatial resolution of $0.64 \mathrm{~mm} /$ pixel. During the experiments the camera was placed under an angle with respect to the window and the model (see Fig.1), in order to avoid any reflections coming from the camera itself to appear in the image.

Before the experiments, the camera was calibrated using a black body simulator. When performing the calibration the germanium window that is mounted in the wind tunnel for optical access is placed between the camera and the black body. In this way the transmissivity of the window is already included in the calibration and does not have to be taken into account afterwards.

Before the wind tunnel was started, the model was heated by means of a $1 \mathrm{~kW}$ lamp that increased the temperature of the plate to approximately $65{ }^{\circ} \mathrm{C}$. As can be seen in section 4.2 , the initial temperature distribution was rather uniform, showing maximum variations of approximately $5 \mathrm{~K}$.

(a)

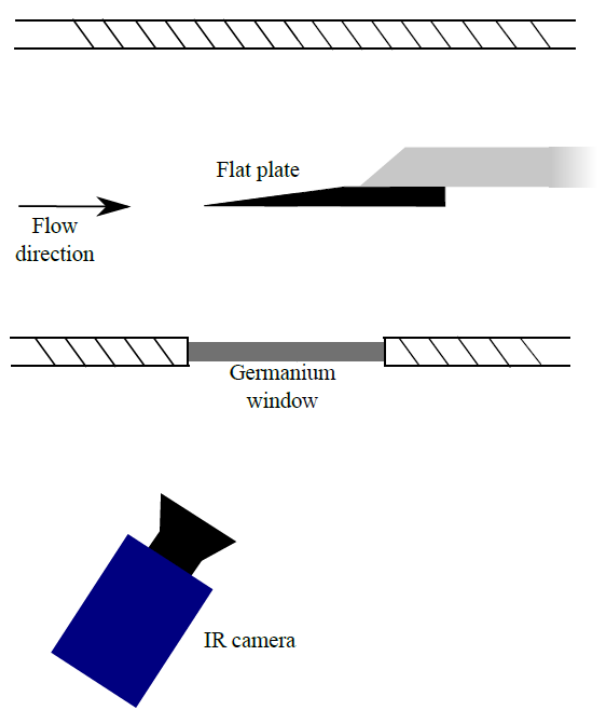

(b)
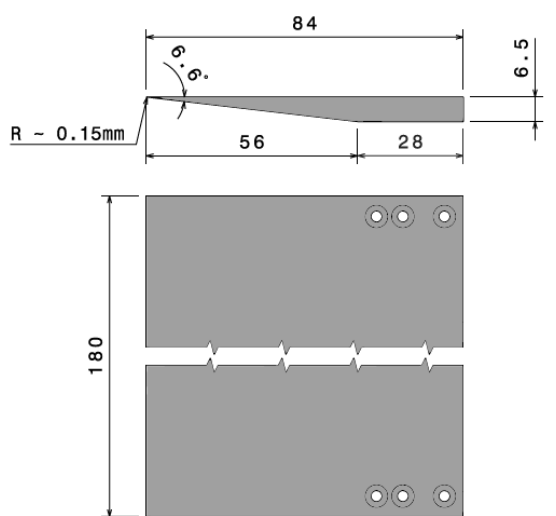

Fig.1. Experimental setup. (a) Sketch of the wind tunnel setup (top view) (b) Dimensions of the model in mm

\section{Data reduction}

As has already been mentioned in the introduction, a turbulent boundary layer has a much higher convective heat transfer compared to a laminar boundary layer. Since the model is heated to a temperature well above the adiabatic wall temperature before the run, the turbulent part of the flat plate cools down faster than the laminar portion. So, in principle it is possible to infer the transition location directly from the temperature distribution. This analysis is, however, complicated by the fact that the flat plate has a non-uniform thickness. The wedge shape (see Fig.1(b)) will cause the thin leading edge region to cool down much faster than the back portion of the plate, even though the flow is laminar. Furthermore, the temperature distribution provides only a delayed representation of the transition location due to a temporal variation in the surface heat flux and the effects of lateral conduction. In order to obtain the instantaneous transition location, it is therefore necessary to directly have access to the value of the convective heat flux.

To obtain the heat flux from the measured temperature distribution in time various data reduction techniques are available, which can be grouped in three classes according to Walter and Scott [2]. Type 1 methods solve the unsteady 1D heat conduction equation in closed form and deliver an analytical solution for the convective heat flux as function of the surface temperature. Well-known examples of this type of method are the approaches from Cook \& Felderman [3] and Kendall \& Dixon [4]. Although robust and fast, these methods assume a 1D semi-infinite surface so it is not possible to take into account the non-uniform thickness of the model nor the effects of lateral conduction. Type 2 methods are more flexible and the convective heat fluxes are derived from a direct numerical solution of the unsteady 2D or 3D heat conduction equation inside a discretized form of the wind tunnel model. Finally, type 3 methods solve the full-inverse problem, usually via an adjoint-type of solution [5], which also provides the sensitivities of the solution, which can be useful in a multi-dimensional analysis of the problem. 
The data reduction method used in this paper can be classified as a type 2 method. The unsteady heat conduction equation is solved in a 2D slice of the flat plate model (see Fig.2). On the top of the flat plate, the measured surface temperatures are applied as time-dependent Dirichlet boundary conditions. Because there is no measurement data available for the bottom side (which is not watched by the camera during the experiments), the same measured temperature signal is also applied here. This approach corresponds to using an adiabatic boundary condition along the centerline of the model. On the rear face of the plate a uniform (in space) time-dependent Dirichlet boundary condition is applied, using the values measured at the trailing edge of the plate. As initial condition, it is assumed that the temperatures measured before starting the tunnel are constant throughout the thickness of the plate. These assumptions are not perfect, since there will be temperature variations between the top and bottom of the plate, both at the start and during the run. However, the goal of calculating the heat fluxes is not to derive quantitative information about the state of the boundary layer (skin friction, thickness etc...), but to get an estimate of the transition location and its behaviour in time.

The 2D heat equation is solved on an unstructured grid with triangular cells, the mesh has 80 cells in y-direction and 1200 cells in x-direction. The surface heat flux is calculated from the temperature gradient at the surface, which is estimated using a second order accurate stencil. Essentially no variations in the calculated surface heat flux were found when using more than 40 cells in y-direction.

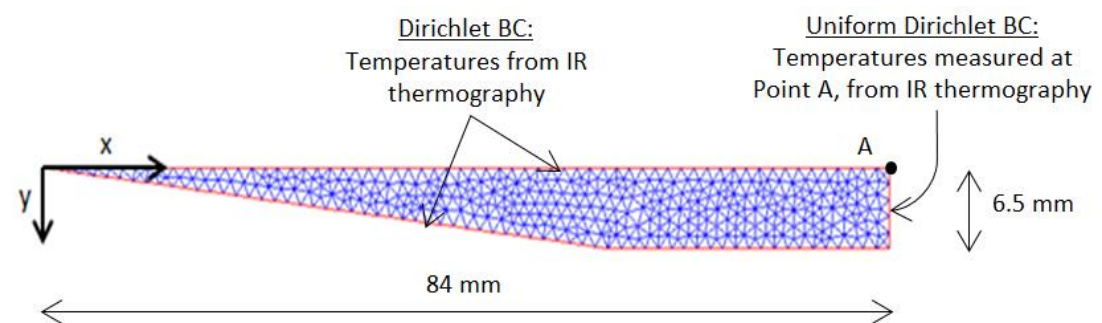

Fig. 2. Coarse version of the mesh used for solving the unsteady heat conduction equation

\section{Results}

As mentioned in the previous section, the analysis of the infrared thermography data involves the calculation of the heat flux distribution along the model's surface. This distribution will be compared to the theoretical heat flux coefficients one would expect for a supersonic compressible laminar/turbulent flat plate boundary layer. The theoretical solutions are discussed in section 4.1 and section 4.2 is devoted to the analysis of the infrared thermography data.

\subsection{Reference solutions}

\subsubsection{Heat flux coefficients for the laminar boundary layer}

For compressible laminar flows it is common practice to estimate the skin friction and heat flux coefficients using the reference temperature method. Following the work of Chapman and Rubesin [6], the skin friction coefficient $C_{f}$ may be approximated as:

$$
C_{f}=\frac{2 \mu_{w} \frac{\partial U(x, 0)}{\partial y}}{\rho_{e} U_{e}{ }^{2}} \approx \frac{0.664 \sqrt{C^{*}}}{\sqrt{R e_{x e}}}
$$

With ${ }_{w}$ the dynamic viscosity of the fluid, $\quad$ the density and $U_{e}$ the mean velocity ( $w$ : at wall \& $e$ : at boundary layer's edge). Furtermore, _ - and $C^{*}$ the Chapman-Rubesin parameter, which is defined as: ( - In this expression $T^{*}$ is the reference temperature at which all fluid properties are evaluated ( , , k etc..). The reference temperature itself is a function, of amongst others, the Mach number at the edge of the boundary layer and wall temperature. In this paper the empirical correlation by Eckert [7] is used: $\quad(\quad-)$. Using the Reynolds analogy, one can now also estimate the heat flux coefficient $C_{h}$ from the skin friction coefficient $C_{f}$ :

$$
C_{h}=\frac{k_{w} \frac{\partial T(x, 0)}{\partial y}}{\rho_{e} U_{e} C_{p e}\left(T_{a w}-T_{w}\right)} \approx \frac{C_{f}}{2 P r^{2 / 3}}
$$

In which $\operatorname{Pr}$ is the Prandtl number, $k_{w}$ the thermal heat conductivity of the fluid at the wall and $T_{a w}-T_{w}$ denotes the difference between the adiabatic wall temperature and the actual wall temperature. In a strict sense this form of the Reynolds analogy only holds for a constant wall temperature. However, as one can see in Fig.3(a) in the current experiments there is a considerable temperature gradient ( $\mathrm{dT} / \mathrm{dx}>1 \mathrm{~K} / \mathrm{mm}$ during the first 5 seconds of the run) in the 
first $\sim 40 \mathrm{~mm}$ from the leading edge. It is therefore doubtful if the Reynolds analogy can be used to provide a good reference solution for comparison for this particular case. Therefore as a next step in the validation process, it was decided to solve the steady compressible laminar boundary layer equations using a finite difference approach. The temperature profile derived from the infrared thermography measurements in this approach is imposed as a wall boundary condition, combined with a no slip condition for the velocity at the wall. The inlet condition is provided by a compressible Blasius boundary layer profile. The effect of compressibility is taken into account by applying the Illingworth transformation to the standard incompressible Blasius profile. The inlet is placed $1 \mathrm{~mm}$ from the leading edge of the plate.

The validity of using the Reynolds analogy in the current experiments is further illustrated in Fig.3. Fig.3(a) shows the temperature profiles that were used as input for the boundary layer solver. In total, seven profiles were considered, an artificial profile representing an isothermal wall at $275 \mathrm{~K}$ and six profiles that were obtained from the infrared thermography measurements $(t=1,5,9,13,17$ and 21 seconds, excluding wind tunnel start-up time of 3 seconds). Fig.3(b) shows the development of the ratio $2 \mathrm{C}_{\mathrm{h}} \mathrm{Pr}^{2 / 3} / \mathrm{C}_{\mathrm{f}}$ over the plate. This ratio directly follows from the Reynolds analogy as it is given in Eq. (2). A perfect agreement with the Reynolds analogy would correspond to a value of 1 in the plot. As one would expect, the isothermal wall case delivers values close to 1 . Both $C_{h}$ and $C_{f}$ deviate less than $3 \%$ from the values calculated by means of the reference temperature method. However, for the experimental temperature profiles the situation is completely different. The skin friction calculated by the boundary layer solver is still in good agreement with Eq. (1) and variations in the skin friction along the length of the model are less than $5 \%$. The heat flux coefficient on the other hand shows a completely different behaviour; along the length of the plate $\mathrm{C}_{\mathrm{h}}$ occasionally deviates more than $50 \%$ from the values that are predicted by the Reynolds analogy.

Clearly, the Reynolds analogy does not hold true for walls having a large streamwise temperature gradient and for a fair comparison with the experimental data, one should compare the experimental heat fluxes with the heat fluxes coming from a laminar boundary layer solver.

(a)

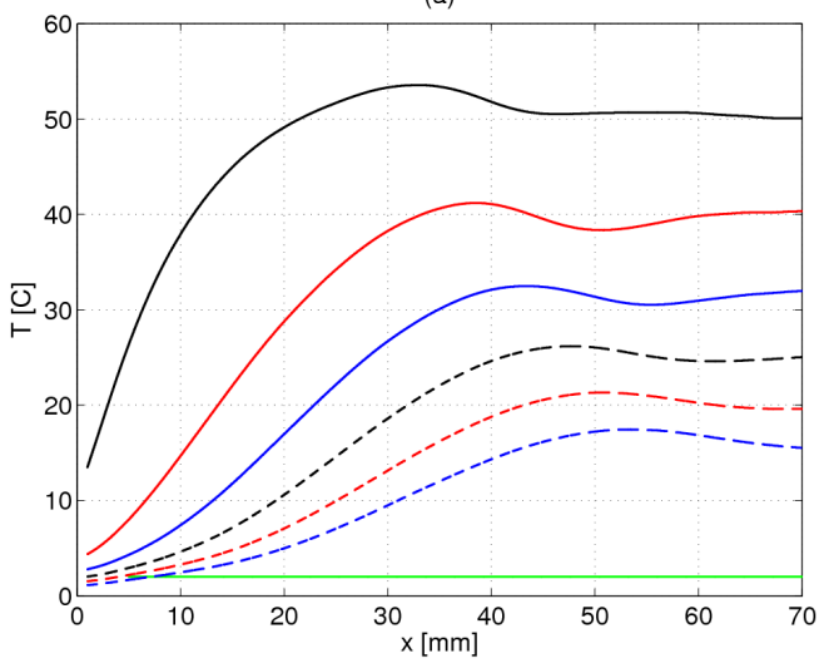

(b)

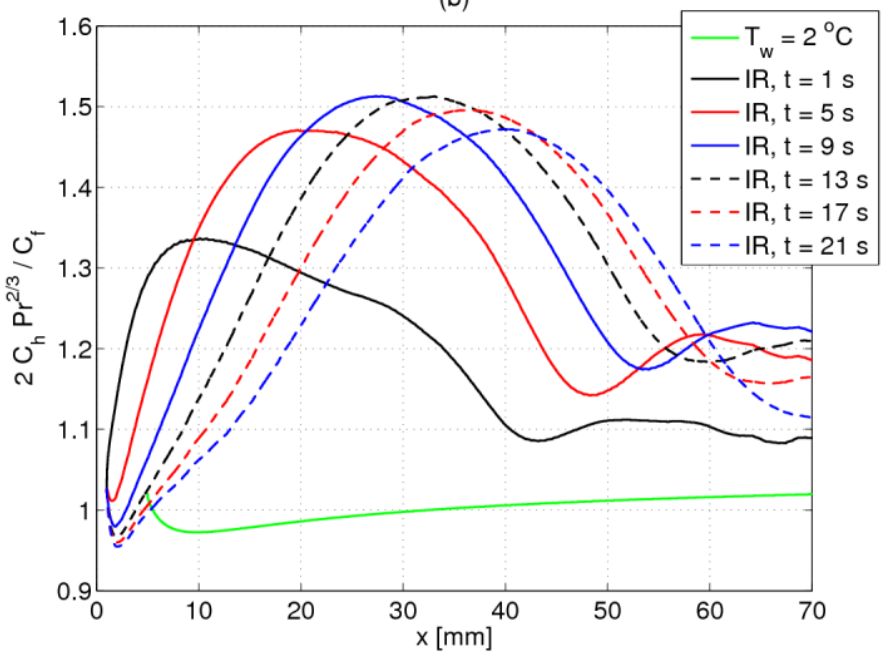

Fig.3. Effects of the plate's temperature distribution on the validity of the Reynolds analogy. (a) Temperature development in time (b) Normalized ratio of the heat flux and skin friction coefficients $\left(2 C_{h} P^{2 / 3} / C_{f}=1\right.$ implies a perfect agreement with the Reynolds analogy)

\subsubsection{Heat flux coefficients for the turbulent boundary layer}

In order to obtain the skin friction coefficient for the turbulent boundary layer, Kármán-Schoenherr's relation [8] is used in combination with the compressibility correction from Spalding and Chi [9]. The skin friction coefficient is then again related to the heat flux coefficient via the Reynolds analogy of Eq. (2). For the laminar case it was shown that the Reynolds analogy is no longer valid because of the high temperature gradient in the streamwise direction. The turbulent portion of the boundary layer, however, falls in the portion of the plate where wall temperature variations are relatively modest (typically less than 5 degrees). Therefore for the turbulent boundary layer the Reynolds analogy is still considered to be a fair assumption for calculating the heat flux coefficients.

\subsection{Transition detection}

The initial temperature distribution before starting the wind tunnel is shown in Fig.4a, which ranges from 60 to $65 \mathrm{C}$. As the wind tunnel is operational, the flow is cooling down the model and Fig.4b shows the temperature distribution after 17 seconds of running time (including approximately 3 seconds of tunnel start-up time). Clearly visible in the image are six $0.2 \mathrm{~mm}$ thick zig-zag strips that have been applied at respectively $20,30,40,50,60$ and $70 \mathrm{~mm}$ from the leading edge. The purpose of these strips is to trigger transition and guarantee a turbulent boundary layer downstream of their 
location. To verify if the strips induce transition or not, Fig.5 shows a comparison between oil flow results and thermography. The oil flow visualization shows the presence of a turbulent spot (wedge) due to an irregularity in the oil film having a spreading rate of approximately 8 degrees. When comparing this to a normalized thermogram, showing the temperature distribution near the edge of the zigzag trip, a similar wedge is observed confirming the presence of transition. On the lower side of the plate there is no strip such that the temperature distribution in this location can be compared with the tripped portion of the plate. From the raw thermogram, already a considerable difference between the upper and lower portions of the plate can be observed. When comparing the measurements at a given streamwise location downstream of the leading edge, it can be noted that for the strips located at 20, 30, and 40 mm the temperature is lower compared to a case without strip (located on the lower side of the plate). In contrast, for a transition strip located at 50,60 or $70 \mathrm{~mm}$, the temperature is not notably different. This suggests that for a clean surface without zigzag strips, transition occurs somewhere between 40 and $50 \mathrm{~mm}$.

(a)
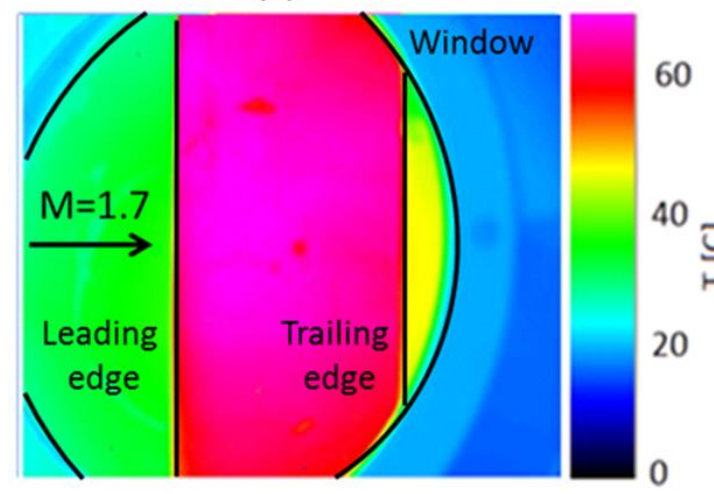

(b)

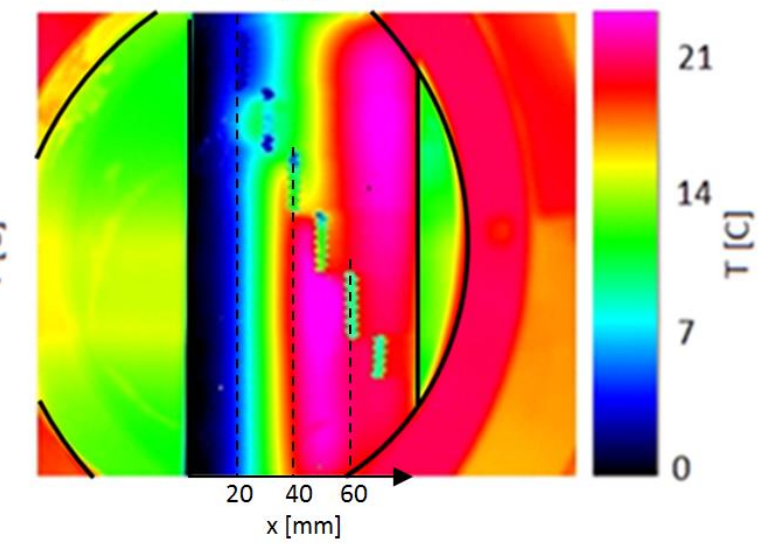

Fig.4. Temperature distribution before starting the tunnel (a) and after running the tunnel for 17 seconds (b)

From the raw thermograms, temperature profiles can be extracted. Fig.6 shows some typical temperature distributions for a unit Reynolds number of $3510^{6} \mathrm{~m}^{-1}$ (a) and $70 \quad 10^{6} \mathrm{~m}^{-1}$ (b). At the location of the transition strip the temperature profiles are blanked because the measured values are considered not to be valid due to the different optical and thermal properties of the strip with respect to the painted stainless steel.

For the case of $\operatorname{Re}_{\infty}=3510^{6} \mathrm{~m}^{-1}$ (Fig.6a) the temperature profile for the clean configuration increases up to approximately $43 \mathrm{~mm}$. From that point on the temperature decreases in streamwise direction and stabilizes again around $59 \mathrm{~mm}$. The thickness of the shock generator increases until $56 \mathrm{~mm}$ from the leading edge and then maintains a constant thickness of $6.5 \mathrm{~mm}$ until the trailing edge (see Fig.6). So, if the complete boundary layer was laminar one would expect the steep temperature increase to continue until $56 \mathrm{~mm}$. The fact that the temperature profile deviates from this trend and begins to decrease already is an indication that the convective heat transfer must increase.

Furthermore, notice that the temperatures downstream of the 40 and $50 \mathrm{~mm}$ trip (where a turbulent boundary layer is present) are lower than those of the clean temperature profile, indicating that for the clean configuration the flow is not yet turbulent at these locations. After $59 \mathrm{~mm}$ the clean and tripped profiles again reach the same temperatures, indicating that the transition process for the clean profile is completed. Based upon these observations, transition is confined to the region between $x=43-59 \mathrm{~mm}$ from the leading edge (centred around $\mathrm{x}=51 \mathrm{~mm}$ ).

The same analysis can also be carried out for the case of having a unit Reynolds number of $\operatorname{Re}_{\infty}=70 \quad 10^{6} \mathrm{~m}^{-1}$, yielding a transition region from $24-32 \mathrm{~mm}$, (centred around $28 \mathrm{~mm}$ ). This corresponds to transitional Reynolds numbers of $1.810^{6}$ and $1.910^{6}$ for the low and high unit Reynolds case, respectively.

(a)

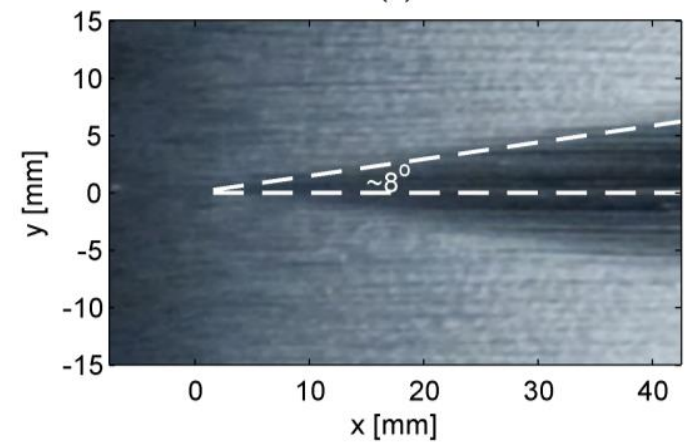

(b)

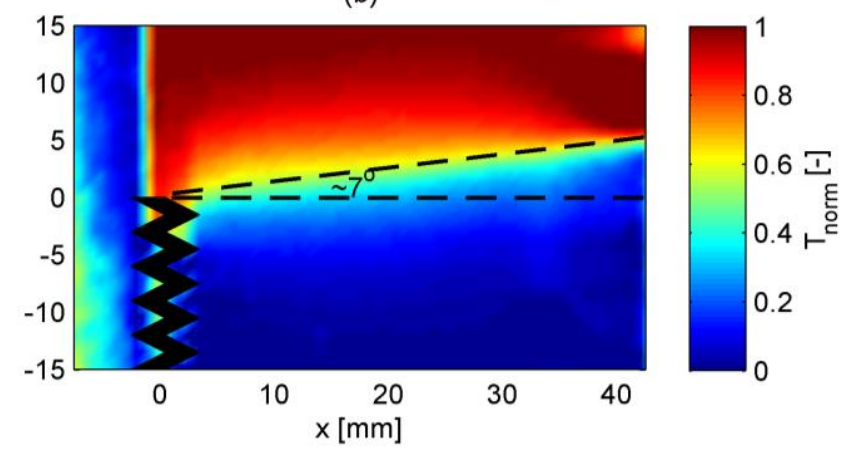

Fig.5. Turbulent wedge development, (a) downstream of a small roughness element, using oil flow visualization (b) downstream of the edge of a zig-zag strip, measured with IR thermography ( 
(a) $-\operatorname{Re}_{\infty}=35 \times 10^{6} \mathrm{~m}^{-1}$

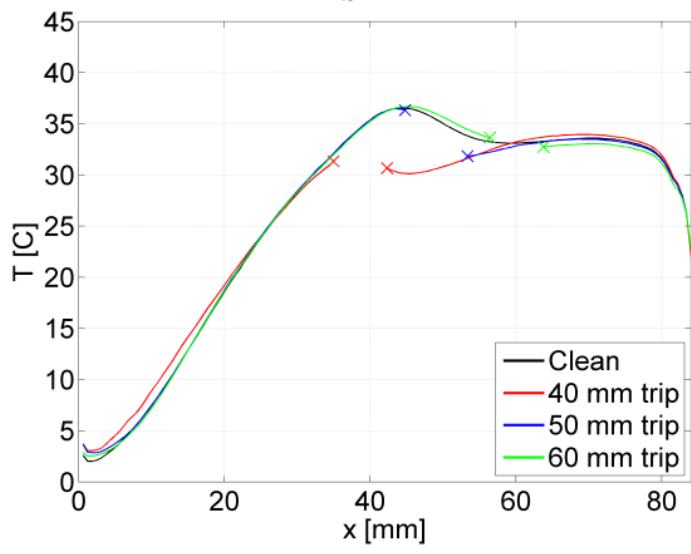

(b) $-\operatorname{Re}_{\infty}=70 \times 10^{6} \mathrm{~m}^{-1}$

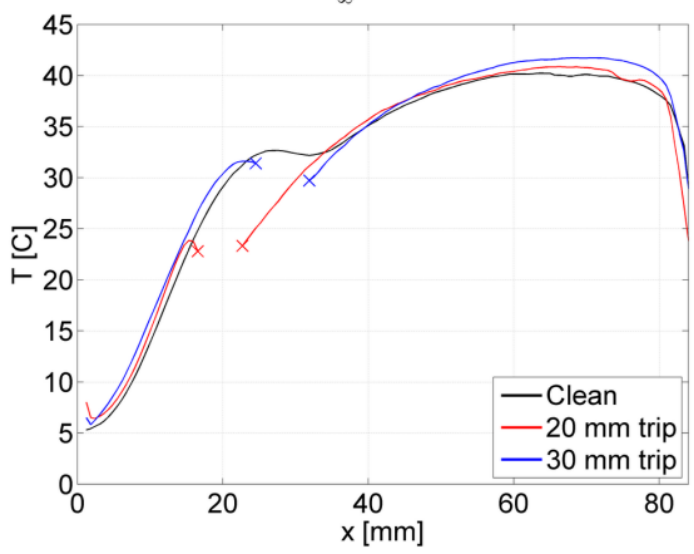

Fig.6. Typical temperature profiles measured for unit Reynolds numbers of $3510^{6} \mathrm{~m}^{-1}$ (a) and $70 \quad 10^{6} \mathrm{~m}^{-1}$ (b)

From the preceding discussion it is clear that the temperature distribution can be very useful in providing a first indication of the transition location. However, as was already noted in section 3 , the temperature distribution is only indirectly affected by the transition location (by means of the surface heat flux). The exact transition location may be modulated by a possible movement of the transition front, varying plate thickness and the effects of lateral conduction. In order to get a better estimate of the transition location, it is necessary to obtain at least a qualitative impression of the convective heat transfer distribution. Therefore, the method outlined in section 3 is used to calculate the heat flux from the temperature measurements.

In Fig.7a the result is shown for such a calculation and it shows the variation of the heat flux coefficient as a function of time and location (for a non-tripped boundary layer). As a reference, the theoretical values are given for a laminar and turbulent boundary layer. Furthermore also the results for the laminar boundary layer code are shown. Close to the leading edge $(\sim 20 \mathrm{~mm})$ a big discrepancy is found between the laminar estimates and the experiment. Whereas theory predicts a decrease in surface heat flux due to boundary layer development, the experiments actually show an increase when moving in the streamwise direction. This can be explained by the fact that at these locations, the leading edge region is extremely thin (thickness is $\sim 2 \mathrm{~mm}$ at $20 \mathrm{~mm}$ ) and therefore cools down very rapidly. During the start-up phase of the wind tunnel already more than 50 degrees is lost, making an accurate determination of the heat fluxes in this region complicated.
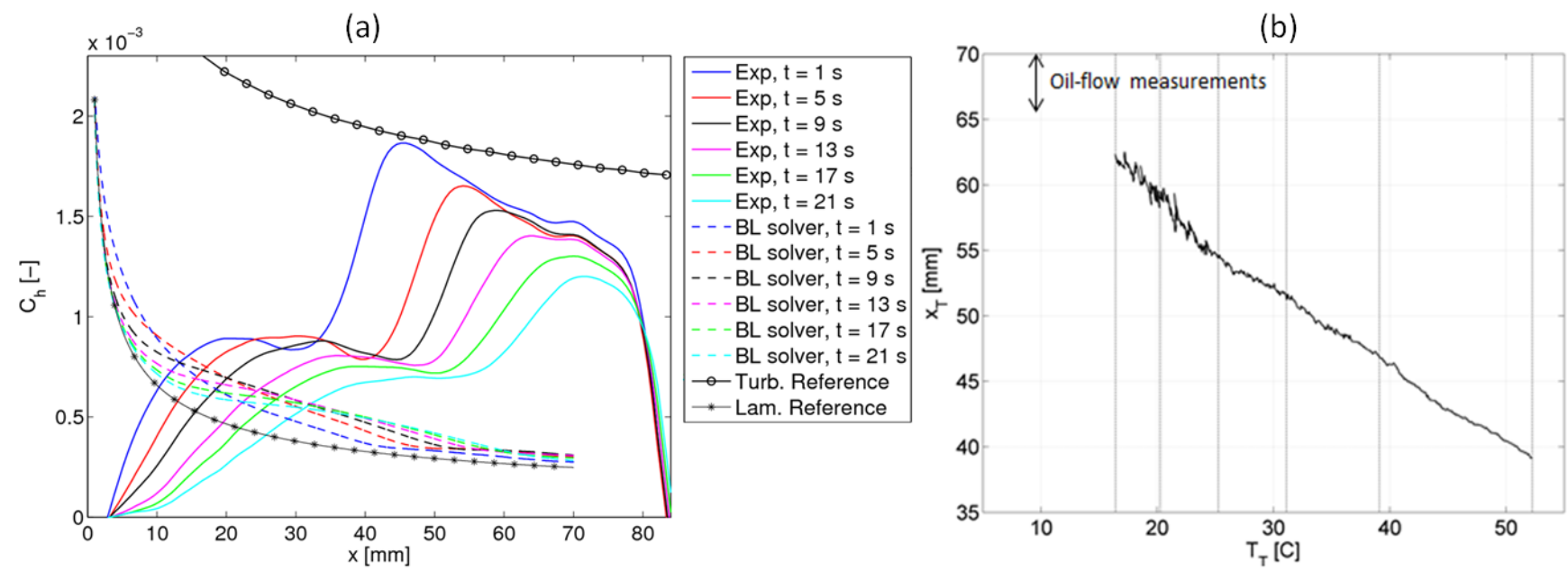

Fig. 7. Heat flux coefficients recorded at various times during the run (a) and the movement of the transition location $x_{T}$ as a function of the local temperature at transition $T_{T}$ (b) The grey vertical lines in (b) correspond to the time instances presented in (a)

Further downstream, the experimental heat flux coefficients can be compared with the solutions from the laminar boundary layer solver. The experimentally determined values in the laminar regime are typically $30-50 \%$ larger than those predicted by the boundary layer solver. This probably is due to the assumption that the temperatures are the same on both the top and bottom of the model when calculating the experimental heat flux coefficients. In practice, however, there will be a temperature difference between the top and bottom surface. Especially, because the flow on the 
bottom surface has to pass an oblique shock wave first, which will increase the mass flow rate in this part. The mass flux $\mathrm{e} \mathrm{U}_{\mathrm{e}}$ is $\sim 15 \%$ higher on the bottom of the model compared to the top. Given the same heat flux coefficients on the top and bottom of the model, this would translate in a higher convective heat flux on the bottom. Therefore this part cools down faster and a temperature gradient between the top and bottom surface will be formed. The top wall will therefore not only lose its heat via the process of convection, but also via the process of heat conduction through the plate. This would explain why the experimentally determined heat flux coefficients are higher than the values following from the boundary layer solver.

The transition region, our main focus of interest, is however clearly visible for all measurement times. As time progresses and the temperature of the plate drops, the transition location clearly moves downstream. Starting at approximately $39 \mathrm{~mm}$ from the leading edge 1 second after the tunnel is fully started and shifting $23 \mathrm{~mm}$ downstream to $62 \mathrm{~mm}$ from the leading edge after 21 seconds of run time. Fig. $7 \mathrm{~b}$ shows the transition location versus the temperature at the transition location, showing a nearly linear relation between both quantities. As a reference, also the transition locations found by oil-flow visualizations for a cold wall $\left(\sim 10^{\circ} \mathrm{C}\right)$ are shown. Both methods are in good agreement with each other, assuming that the linear trend determined by infrared thermography can also be extrapolated to lower temperatures.

\section{Conclusions}

Infrared thermography measurements were performed on flat plate boundary layer transition in a supersonic flow $\left(M=1.7\right.$ and $\left.R_{\infty}=3510^{6} \mathrm{~m}^{-1}\right)$. The plate was heated uniformly before the wind tunnel run to a temperature of $\sim 65^{\circ} \mathrm{C}$. During the course of the wind tunnel run, the plate cools down rapidly, stabilizing the laminar boundary layer and causing a moving transition front. The instantaneous transition locations are derived from the surface heat flux distribution. The heat fluxes are calculated by solving the unsteady heat conduction equation in a $2 \mathrm{D}$ slice of the model, with the temperatures recorded by the infrared camera imposed as time-dependent Dirichlet boundary conditions.

A near-linear relation is found between the transition location and the temperature at the transition location $\left(\sim 0.6 \mathrm{~mm} /{ }^{\circ} \mathrm{C}\right)$. The transition front moves from $\mathrm{x}=39 \mathrm{~mm}$ at the start of the run to $\mathrm{x}=62 \mathrm{~mm}$ at the end of the run. This corresponds to a Reynolds number range of $\mathrm{Re}_{\infty}=1.4-2.210^{6}$. The linear trend that is found is shown to be in good agreement with oil-flow visualizations that were performed on the flat plate (assuming that the linear trend can be extrapolated to the lower temperatures at which the oil-flow visualization was performed).

These results show that infrared thermography can be used as a tool for recording a moving transition front and also highlight the importance of knowing the wall temperature when performing transition measurements.

\section{REFERENCES}

Mack, L.M., "Boundary Layer Stability Theory”, pp. 3-1 - 3-81 of AGARD, 1984

Walker D.G. and Scott E.P., "Evaluation of Estimation Methods for High Unsteady Heat Fluxes from Surface Measurements", Journal of Thermophysics and Heat Transfer, vol. 12, no. 4, pp. $543-551,1998$

Cook W.J. and Felderman E.J., "Reduction of Data from Thin-Film Heat-Transfer Gages: A Concise Numerical Technique". AIAA Journal, vol. 4, no.3, pp. 561 - 562, 1966

[4] Kendall D.N., Dixon W.P. and Schulte E.H., "Semiconductor Surface Thermocouples for Determining Heat Transfer Rates". IEEE Transactions on Aerospace and Electronic Systems, vol. AES-3, no. 4, 1967.

zisik M.N. and Orlande H.R.B, "Inverse Heat Transfer - Fundamentals and Applications". Taylor \& Francis, New York, 2000

[6] Chapman, D.R. and Rubesin, M.W., "Temperature and velocity profiles in the compressible laminar boundary layer with arbitrary distribution of surface temperature", Journal of Aeronautical Sciences, vol.16, pp. 547-565

[7] Eckert, E.R.G., Engineering relations for friction and heat transfer to surfaces in high velocity flow, Journal of Aeronautical Sciences, vol. 22, pp. 585-587, 1955

[8] Schoenherr, K.E., Resistance of plates, Trans. Soc. Nav. Architects Mar. Eng., vol. 40, 1932

[9] Spalding, D.B. and Chi, S.W., The drag of a compressible turbulent boundary layer on a smooth flat plate with and without heat transfer, Journal of Fluid Mechanics, vol. 18, pp. 117-143, 1964 